\title{
Exploiting Inertia of Wind Turbines in Power Network Frequency Control: A Model Predictive Control Approach
}

\author{
N.P.G. van Deelen, A. Jokić, P.P.J. van den Bosch and R.M. Hermans
}

\begin{abstract}
With the expected increase in penetration level of wind turbine generators in the near future, it will be necessary for them to participate in power network frequency control. In this paper we exploit the inertia of wind turbine generators using model predictive control (MPC). In this way wind turbines can actively contribute to primary control. Safe operation is possible because MPC explicitly takes safety constraints into account. In a case study a nonlinear model of a power network is balanced by exploiting the inertial response of wind turbine generators. We have considered both centralized MPC and a decentralized MPC implementation, and have shown their efficiency in counteracting deviations in generation and demand introduced either by unpredictable exogenous disturbances, or by imbalanced transients during market rescheduling processes. The obtained results demonstrate the potential of wind turbine inertia exploitation in contributing to the challenging task of balancing future power networks.
\end{abstract}

\section{INTRODUCTION}

In electrical power systems, supply and demand have to match in real-time due to the fact that electrical energy cannot be stored efficiently in large amounts. A mismatch between supply and demand leads to frequency deviation in the power grid. If not accounted for, these frequency deviations might lead to instability and load shedding [1], [2]. With the ongoing penetration of renewable energy sources in the power production, the amount of generators responsible for the power production is increasing significantly. With the increase of distributed generation electrical power networks become increasingly complex. Furthermore, the power supply becomes less predictable due to intermittent energy sources such as wind and sun. Moreover, most of the renewable energy sources are not involved in frequency regulation. This means that renewable energy sources introduce large uncertainties, and on the other hand do not participate in stabilization of the power network. With a significant penetration of renewable energy sources expected in the near future this may introduce instability problems into the network. At a point in the future, conventional generators will no longer be able to account anymore for sudden frequency fluctuations. At the same time, liberalization of the energy market has contributed to a significant change in the way power generation can be controlled. Energy is traded on the Power-Exchange market, which is driven by competitive

This work is supported by the E-PRICE project (Price-based Control of Electrical Power Systems) funded by the European Commissions Seventh Framework Programme (Theme ICT for energy efficiency).

N.P.G. van Deelen, A. Jokić, P.P.J. van den Bosch and R.M. Hermans are with the Department of Electrical Engineering, Control Systems Group, Eindhoven University of Technology, P.O. Box 513, 5600 MB Eindhoven, The Netherlands, Corresponding E-mail address: A. Jokic@tue.nl.

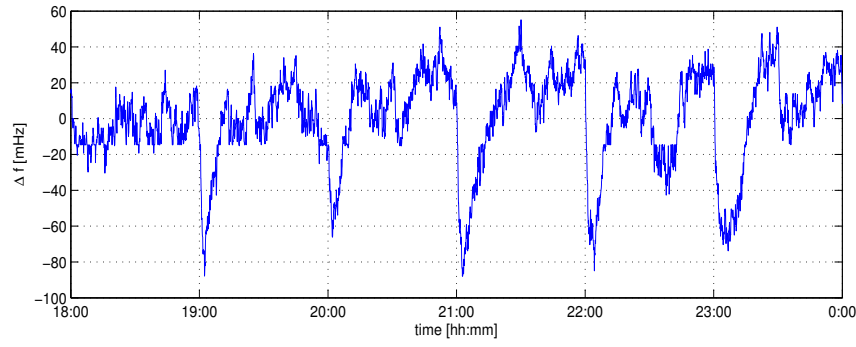

Fig. 1. Frequency measurement in the EU power grid. Large frequency deviations occur at full hour boundaries.

economic forces. The result of this is that the system is often being pushed towards its stability boundaries [1]. This is reflected in increased frequency fluctuations at the hourly boundaries in the European power network. Figure 1 shows frequency measurement during evening hours (performed on the European (ENTSO-E) grid [3]). Recent studies have shown that the consequences of the development of the electrical energy markets in the European countries, in combination with the continuous increase in market participant activities, are already visible today and have a large impact on daily grid operation [1], [2]. Frequency deviations are getting larger and more frequent. A large part of reserves (up to 75\%), intended for use in case of system failures, is being employed during regular network operation, as a consequence of the economic settlement procedure.

Wind power is the most rapidly increasing renewable energy source [4]. To further facilitate increased levels of wind generation, it might be necessary for the wind turbines to contribute to the system's control by exploiting their inertial response. Possible solutions for the lack of inertial response in variable speed wind turbines have already been presented in e.g. [5], [6], [7] and some of the references therein.

Model predictive control (MPC) has proven to be highly efficient in the process industry. Multiple studies have indicated that MPC has a potential to tackle problems facing the future power networks [8], [9], [10]. The unique feature of MPC is its ability to take constraints explicitly into account. Therefore it could be very efficient in fully and optimally exploiting the inertial response of wind turbine generators. The main goal of this work is to investigate and present these potentials of MPC controlled wind turbines in their support to real-time power network frequency control.

\section{WIND TURBINE GENERATOR DYNAMICS}

The dynamics of a wind turbine generator used in this paper are represented as a two-mass model connected to the 
power grid through a back-to-back full converter bridge, as shown in Figure 2. The differential equations of the wind turbine generator system are given by [11]:

$$
\begin{aligned}
\frac{\mathrm{d} \omega_{h}}{\mathrm{~d} t} & =\frac{1}{J_{h}}\left(\frac{P_{\text {aero }}}{\omega_{h}}-k K_{s} \theta_{\mathrm{diff}}-D_{h} \omega_{h}\right), \\
\frac{\mathrm{d} \theta_{\mathrm{diff}}}{\mathrm{d} t} & =k \omega_{h}-\omega_{g}, \\
\frac{\mathrm{d} \omega_{g}}{\mathrm{~d} t} & =\frac{1}{J_{g}}\left(K_{s} \theta_{\mathrm{diff}}-\frac{P_{\mathrm{gen}}}{\omega_{g}}-D_{g} \omega_{g}\right), \\
\frac{\mathrm{d} v_{\mathrm{dc}}}{\mathrm{d} t} & =\frac{T_{\mathrm{gen}} \omega_{g}-P_{\mathrm{ref}}}{C_{\mathrm{dc}} v_{\mathrm{dc}}} .
\end{aligned}
$$

The prevailing wind delivers a power $P_{\text {aero }}[\mathrm{W}]$ to the rotor blades of the wind turbine. This makes the turbine's hub rotate with a hub speed $\omega_{h}[\mathrm{rad} / \mathrm{s}] . \theta_{\text {diff }}[\mathrm{rad}]$ is the deformation of the shaft, while $K_{s} \theta_{\text {diff }}[\mathrm{Nm}]$ is the torque on the drive shaft with gear ratio $k$ and spring constant $K_{s}$ $[\mathrm{Nm} / \mathrm{rad}] . \omega_{g}[\mathrm{rad} / \mathrm{s}]$ is the speed of the generator. The inertia of the hub is $J_{h}\left[\mathrm{kgm}^{2}\right]$ and the inertia of the generator is $J_{g}\left[\mathrm{kgm}^{2}\right] . D_{h}\left[\mathrm{kgm}^{2} / \mathrm{s} / \mathrm{rad}\right]$ and $D_{g}\left[\mathrm{kgm}^{2} / \mathrm{s} / \mathrm{rad}\right]$ are the damping of the rotor and generator shaft, respectively. The full converter bridge dynamics are given by (1d), which has two independent control inputs: $P_{\text {ref }}[\mathrm{W}]$, the power which will be delivered to the grid and $P_{\text {gen }}[\mathrm{W}]$, the power input of the generator. $v_{\mathrm{dc}}[\mathrm{V}]$ is the voltage at the capacitor and $C_{\mathrm{dc}}[\mathrm{F}]$ is the capacitance of the converter bridge.

$P_{\text {aero }}$ in (1a) is a function of hub speed $\left(\omega_{h}\right)$ and wind speed $\left(v_{w}[\mathrm{~m} / \mathrm{s}]\right)$ [12], as follows: $P_{\text {aero }}\left(\omega_{h}, v_{w}\right)=$ $\frac{1}{2} \rho A C_{p}(\lambda) v_{w}^{3}, \lambda=\frac{R \omega_{h}}{v_{w}}$, where $\rho\left[\mathrm{kg} / \mathrm{m}^{3}\right]$ is the air density; $A\left[\mathrm{~m}^{2}\right]$ is the area covered by the rotor; $C_{p}$ is the power coefficient of the turbine blades; $R$ [m] is the radius of the rotor and $\lambda$ is the tip speed ratio.

The time constant of the converter bridge is only one tenth of the sample time of 1 second (for details on discretization see Section IV), which means that we can simplify the model by neglecting the dynamics in (1d). As a consequence, only one control input of the wind turbine generator remains.

Note that the expressions (1a) and (1c) are nonlinear. We will use a linearization by taking a first order Taylor expansion of the two expressions. The complete linearized version of the wind turbine generator model is summarized as follows:

$$
\begin{aligned}
\frac{\mathrm{d} \omega_{h}}{\mathrm{~d} t} & =\frac{1}{J_{h}}\left(\frac{1}{\bar{\omega}_{h}} \Delta P_{\text {aero }}-\left(\frac{\bar{P}_{\text {aero }}}{\bar{\omega}_{h}^{2}}+D_{h}\right) \Delta \omega_{h}-k K_{s} \Delta \theta_{\text {diff }}\right), \\
\frac{\mathrm{d} \theta_{\mathrm{diff}}}{\mathrm{d} t} & =k \Delta \omega_{h}-\Delta \omega_{g}, \\
\frac{\mathrm{d} \omega_{g}}{\mathrm{~d} t} & =\frac{1}{J_{g}}\left(K_{s} \Delta \theta_{\text {diff }}-\frac{1}{\bar{\omega}_{g}} \Delta P_{\text {ref }}+\left(\frac{\bar{P}_{\text {ref }}}{\bar{\omega}_{g}^{2}}-D_{g}\right) \Delta \omega_{g}\right) .
\end{aligned}
$$

The parameters with bars represent steady state values at which the linearization is performed. The $\Delta$ 's indicate that we are dealing with deviations from the steady state values.

Safety constraints. For safe operation of a wind turbine generator it is important to stay within certain safety boundaries [13]. Each wind turbine generator has a

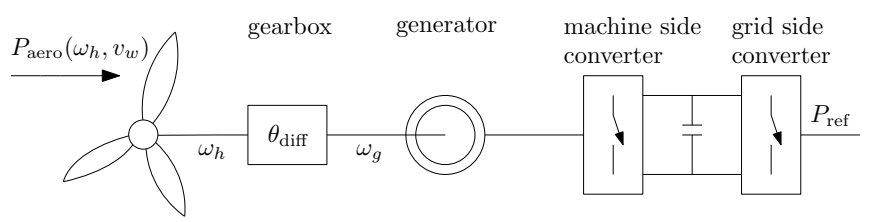

Fig. 2. Block diagram of the wind turbine generator model.

rated power $P_{\text {rated, }}$ which should not be exceeded, i.e. $P_{\text {ref }} \leq P_{\text {rated }}$. Apart from its rated power, each wind turbine generator also has a rated speed $\omega_{h}^{\text {rated }}$. This is an upper bound on the hub speed of the wind turbine. There is also a lower bound on the hub speed, which is given by the cut-in speed of the wind turbine. The operating range of the hub speed of a wind turbine generator is thus given by:

$$
\omega_{h}^{\text {cut }- \text { in }} \leq \omega_{h} \leq \omega_{h}^{\text {rated }}
$$

Another constraint involves the shaft and the gearbox. The shaft deformation angle $\theta_{\text {diff }}$ is allowed to deviate at most $15 \%$ around its nominal value.

\section{A. Inertial response of a wind turbine}

Large rotating blades of a wind turbine contain a large amount of kinetic energy, which can be used to compensate for fast frequency fluctuations in the power network. The amount of energy which can be extracted from the wind turbine generator, i.e. the energy stored in the rotating inertia, is given by:

$$
E=\frac{1}{2}\left(J_{g}+\frac{J_{h}}{k^{2}}\right) \omega_{g}^{2} .
$$

The time the wind turbine can provide power by only using the energy stored in its rotating mass is limited to a short period of about 2-9 seconds [5], [7]. By extracting power from the rotating inertia, the wind turbine slows down. After this period the energy extracted from the generator should be less than the energy extracted from the prevailing wind to speed up the turbine so it will return in its optimal operating point again. The time for regaining the energy can be significantly longer than the time for the energy extraction, giving other generators with slower dynamics sufficient time to take care of the extra power generation.

Wind turbine generators have two operating modes; below and above rated wind speed. In both modes it is possible to temporarily extract a surplus of power from the wind turbine. In above rated wind speed conditions, wind turbine power must be limited in the steady state to the rated value.

\section{Model Predictive Control}

For predictions in the Model Predictive Control (MPC) calculations in this paper we use linear time invariant discrete time models to represent the controlled system. The model is represented in a state space realization of the form:

$$
x(k+1)=A x(k)+B u(k),
$$

where $A \in \mathbb{R}^{n \times n}, B \in \mathbb{R}^{n \times m}, x(k) \in \mathbb{R}^{n}$ and $u(k) \in \mathbb{R}^{m}$.

The MPC controller is based on the outcome of an optimization problem to determine the optimal input. The 
optimization problem, which will be solved every sampling instant is, formulated as a quadratic program of the form:

$$
\begin{array}{rl}
\min _{U} & V(x, U), \\
\text { subject to } & A_{\text {ineq }} U \leq B_{\text {ineq }},
\end{array}
$$

and (5), where the cost function is given by:

$$
V(x, U)=x_{N}^{\top} P x_{N}+\sum_{k=0}^{N-1}\left(x_{k}^{\top} Q x_{k}+u_{k}^{\top} R u_{k}\right) .
$$

The matrices $Q=Q^{\top} \succeq 0, R=R^{\top} \succ 0$ are performance weights, which can be tuned for desired performance. $P=$ $P^{\top} \succ 0$ is the terminal state weight, which is, in addition to terminal state constraints, see e.g. [14] for details, used to guarantee closed loop stability. The computed control inputs are stacked into the vector $U=\left(u^{\top}(0), \ldots, u^{\top}(N-1)\right)^{\top}$, where $N$ is the prediction horizon. Matrices $A_{\text {ineq }}$ and $B_{\text {ineq }}$ are inequality constraint matrices, capturing input and / or state constraints.

The control and state sequences have to satisfy: $u(k) \in \mathbb{U}$, $x(k) \in \mathbb{X}$, where $\mathbb{U}$ is a convex, compact subset of $\mathbb{R}^{m}$ and $\mathbb{X}$ a convex, closed subset of $\mathbb{R}^{n}$. Asymptotic stability can be guaranteed by choosing an appropriate terminal set $X_{f} \subset$ $\mathbb{X}$ and a terminal weight $P$, which satisfies the Lyapunov equation $^{1} P=A^{\top} P A+Q$ [14].

\section{A. Decentralized MPC}

The control of large scale systems, such as power networks, is impossible to implement on one centralized controller. Large scale systems often consist of several subsystems, which have coupled dynamics. In decentralized model predictive control (DMPC), the total system is decoupled into independently controlled subsystems [15]. Again the system to be controlled is described by (5). In DMPC the large scale system is partitioned into multiple subsystems and a controller is designed for each subsystem individually.

Let $M$ be the number of subsystems in the network. We define $x^{i} \in \mathbb{R}^{n_{i}}, i \in \mathfrak{I}:=\{1, \ldots, M\}$, as the vector collecting the state component of the subsystem $i$. Matrix $W_{i} \in \mathbb{R}^{n \times n_{i}}$ collects the $n_{i}$ columns of the identity matrix of order $n$ corresponding to the states, i.e. $x^{i}$ the state of subsystem $i$ is given by $x^{i}=W_{i}^{\top} x$.

We define $u^{i} \in \mathbb{R}^{m_{i}}$ as the vector collecting the inputs corresponding to the inputs of subsystem $i$. Matrix $Z_{i} \in$ $\mathbb{R}^{m \times m_{i}}$ collects the $m_{i}$ columns of the identity matrix of the order $m$ selecting the inputs, i.e. we have the following expression: $u^{i}=Z_{i}^{\top} u$. The local subsystem $i$ is now represented by:

$$
x^{i}(k+1)=A_{i} x^{i}(k)+B_{i} u^{i}(k),
$$

where $A_{i}=W_{i}^{\top} A W_{i}, B_{i}=W_{i}^{\top} B Z_{i}$. Note that it is possible to assign $x$ and $u$ to more than one $x^{i}$ and $u^{i}$

\footnotetext{
${ }^{1}$ Note that this holds only for open loop asymptotically stable systems. In a more general setting, the matrix $P$ and state feedback matrix $K$, obtained as a solution of the appropriately defined Ricatti equation, are used in the so-called dual mode control.
}

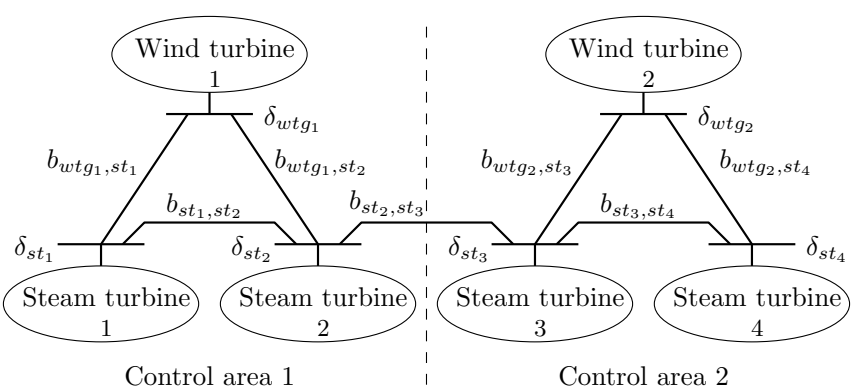

Fig. 3. Schematic representation of a power network consisting of two control areas with three generators in each area.

respectively. This means that there can exist overlap in the definition of the subsystems.

Using (8) for prediction in each local controller, the optimization problem solved for each subsystem is:

$$
\begin{aligned}
\min _{U_{i}} & V_{i}\left(x^{i}, U_{i}\right), \\
\text { subject to } & A_{\text {ineq }}^{i} U_{i} \leq B_{\text {ineq }}^{i} .
\end{aligned}
$$

Here the cost function is given by:

$$
V_{i}\left(x^{i}, U_{i}\right)=x_{N}^{i}{ }^{\top} P_{i} x_{N}^{i}+\sum_{k=0}^{N-1}\left(x_{k}^{i}{ }^{\top} Q_{i} x_{k}^{i}+u_{k}^{i}{ }^{\top} R_{i} u_{k}^{i}\right),
$$

where $Q_{i}=W_{i}^{\top} Q W_{i}, R_{i}=Z_{i}^{\top} R Z_{i}$. For the above presented decentralized MPC scheme, an a posteriori stability check has been presented in [15].

\section{CASE STUdY}

The power network used in the case studies consists of two control areas, which contain three generators each (Figure 3). In each area, one of the generators is a wind turbine and the other two generators are steam turbines. There are two Balance Responsible Parties ${ }^{2}$ (BRP), where each BRP is responsible for the power generation in only one control area and a transmission system operator (TSO) is responsible for the system stability. This research has focused on frequency deviation minimization by optimization-based real-time control, with the aim to exploit the inertial response of wind turbine generators. The wind speed is kept constant in the simulations, as our focus is on the benefits of using the inertial response of the wind turbine in support of the network frequency control.

We use the wind turbine generator model as presented in Section II. To complete the dynamical model of the overall power network from Figure 3, we need the appropriate models of the steam turbine generators and mathematical formulation of generators interconnections. This is done in the following subsections.

Steam turbine generator model. The linearized model

\footnotetext{
${ }^{2} \mathrm{~A}$ BRP is a market agent trading on the power exchange and ancillary service markets.
} 
of the steam turbines is [16]:

$$
\begin{aligned}
\frac{\mathrm{d} \Delta \delta_{i}}{\mathrm{~d} t} & =\Delta \omega_{i}, \\
\frac{\mathrm{d} \Delta \omega_{i}}{\mathrm{~d} t} & =\frac{1}{J_{i}}\left(\Delta P_{M_{i}}-D_{i} \Delta \omega_{i}-\sum_{j \in \mathcal{N}_{i}} \Delta P_{\mathrm{tie}}^{i j}-\Delta P_{L_{i}}\right), \\
\frac{\mathrm{d} \Delta P_{M_{i}}}{\mathrm{~d} t} & =\frac{1}{\tau_{T_{i}}}\left(\Delta P_{V_{i}}-\Delta P_{M_{i}}\right), \\
\frac{\mathrm{d} \Delta P_{V_{i}}}{\mathrm{~d} t} & =\frac{1}{\tau_{G_{i}}}\left(\Delta P_{\text {ref }_{i}}-\Delta P_{V_{i}}-\frac{1}{R_{i}} \Delta \omega_{i}\right) .
\end{aligned}
$$

The $\Delta$ 's indicate that we are dealing with the deviations from the nominal value of the corresponding system's state. The rotor speed of the turbine is represented by $\Delta \omega_{i}[\mathrm{rad} / \mathrm{s}]$, while $\Delta P_{M_{i}}[\mathrm{~W}]$ is the mechanical power, which drives the generator. In a steam turbine generator, the main steam supply is controlled using valves. In the above model, $\Delta P_{V_{i}}$ [W] denotes the valve position, while $\Delta P_{L_{i}}$ [W] denotes the generator load, i.e. the actual power delivered to the consumers. The tie line power flow from bus $i$ to bus $j$ is represented by $\Delta P_{\text {tie }}^{i j}$ [W]. $\mathcal{N}_{i}$ is the set of tie line connected neighboring generators $j$ to generator $i$.

Power network interconnections. The power flow in the tie lines connecting the generators is given by:

$$
\begin{aligned}
& \Delta P_{\text {tie }}^{i j}=b_{i j}\left(\Delta \delta_{i}-\Delta \delta_{j}\right), \\
& \Delta P_{\text {tie }}^{j i}=-\Delta P_{\text {tie }}^{i j},
\end{aligned}
$$

where $\Delta \delta_{i}$ denotes relative voltage phase angle at the generator bus $i$. The phase angle of a steam turbine generator is dependent on its generator frequency. However, the phase angle of a wind turbine generator is independent of the wind turbine generator frequency, because the generator is decoupled from the grid by a converter bridge. The phase angle of the wind turbine generator can be arbitrarily chosen in order to control the power of the turbine injected into the grid. The value of the voltage phase angle $\delta_{\mathrm{wtg}_{1}}$ at wind turbine generator 1 of the power network in Figure 3 is given by:

$$
\delta_{\mathrm{wtg}_{1}}=\frac{P_{\mathrm{ref}, \mathrm{wtg}_{1}}+b_{\mathrm{st}_{1}, \mathrm{wtg}_{1}} \delta_{\mathrm{st}_{1}}+b_{\mathrm{st}_{2}, \mathrm{wtg}_{1}} \delta_{\mathrm{st}_{2}}}{b_{\mathrm{st}_{1}, \mathrm{wtg}_{1}}+b_{\mathrm{st}_{2}, \mathrm{wtg}_{1}}} .
$$

For the centralized MPC controller, the total system (Fig. 3) is lumped into one single model. This model is discretized using zero-order hold on the inputs, and a sample time of 1 second. Simulations are carried out on the continuous time nonlinear model in Simulink.

\section{A. MPC for a power network}

The MPC controller has to divide the requested power among the different types of generators for a change in reference set point, e.g. for a transition from the current to the next Programm Time Unit ${ }^{3}$ (PTU). The knowledge of the dynamics of the different types of generators is used by the MPC controller for this division. The MPC controller uses

\footnotetext{
${ }^{3} \mathrm{PTU}$ is time interval on which electrical energy is traded on energy markets, i.e. amount of energy exchange between market parties is defined per PTU.
}

the outcome of an optimization problem (5), (6), which is solved at every sampling instant. Nonzero elements in the $Q$ matrices penalize the state deviations, while inputs are penalized by choosing nonzero elements in the $R$ matrices. In our problem setting, we penalize the generator frequency deviations of the steam turbines $\left(\omega_{\mathrm{st}_{\mathrm{i}}}\right)$ and the wind turbines $\left(\omega_{g_{i}}\right)$. The control inputs $P_{\text {ref, } w t g_{i}}$ for the wind turbine generators and $P_{\text {ref,st }}$ for the steam turbine generators are also penalized. The power demand $P_{\mathrm{L}, \mathrm{st}_{\mathrm{i}}}$ on each steam turbine can be seen as an uncontrollable, exogenous input.

The inequality matrices $A_{\text {ineq }}$ and $B_{\text {ineq }}$ define the constraints. For safe operation of a wind turbine generator, the upper and lower limit given in (3) are chosen $10 \%$ below $\omega_{h}^{\text {rated }}$ and $10 \%$ over $\omega_{h}^{\text {cut }- \text { in }}$ respectively [12]. Because MPC explicitly takes constraints into account, it is possible to relax these safety margins. It is supposed that the wind turbine generators are producing $200 \mathrm{~kW}$ under rated power. The steam turbine generators are constrained to produce $500 \mathrm{~kW}$ above and below nominal power. Furthermore, the shaft deformation angle $\theta_{\text {diff }}$ is allowed to deviate at most $15 \%$ around its nominal value.

Because of the complexity of large scale power networks it is impossible to use a centralized MPC controller. Another difficulty is that, in the competitive market environment, BRPs are not willing to share their internal and confidential data. For practical implementation of a MPC controlled power network, there is a need for a distributed solution. Multiple studies have alredy been carried out in this area using several approaches, see e.g. [9], [10] and the references therein. The distributed solution used in this work is the DMPC, introduced in [15], where we make use of the overlapping models. More precisely, in case of the DMPC, the states of area $1\left(x^{1}\right)$ are extended with the delta of steam turbine $3\left(\delta_{\mathrm{st}_{3}}\right)$, i.e. with the voltage phase angle of the generator at the interconnection between the two areas. There is a need for this overlap between the systems to be able to control the tie line power flow between the two areas. Tie lines are always accompanied by communication lines, making the communication between neighboring generators possible. In our control scenario, the area 1 will be held responsible for the control of the tie line power flow. The optimization problem, which has to be solved for each subsystem $i$ is formulated in (8) and (9).

\section{B. Classical power network control}

To show the benefit of using MPC and the inertial response of the wind turbine generator, we have made a comparison with current situation, based on conventional Automatic Generation Control (AGC) [16]. Each control area in a power network is equipped with its own AGC controller, i.e. in our case we have two AGC controllers. In the network setup from Figure 3, we take the frequency of steam turbine generator 2 as a reference for the AGC of area 1, and we take the frequency of steam turbine generator 3 as a reference for the AGC of area 2. The AGC controllers are also responsible for the tie line power flow, which is flowing between the areas. 
A BRP requests a certain contribution from each generator involved in frequency regulation. The advantage of MPC over AGC is that the MPC controller has knowledge about the different dynamics of each generator, and exploits this knowledge to obtain optimal dynamical response of the system. As a result of the MPC optimization procedure, the total demanded power will, during the transient periods, be optimally divided among the generators, while in the case of AGC, this division will be based on some predetermined values. Another big advantage of MPC is that it is able to anticipate a priori known events. PTU production programs are known day ahead and therefore it is possible to include them in the predictions used by the MPC controller.

\section{Simulation results}

The parameter values, used in simulations, of the steam turbine generator model (11) and the tie lines (12) are taken from [8]. The parameter values of the wind turbine generator (2) originate from [17].

First we consider a load disturbance, which occurs at time instant $t=50$ seconds, and which lasts for 10 seconds. The disturbance acts as a stepwise load increase of $200 \mathrm{~kW}$ at the bus where the steam turbine 2 in area 1 is connected. At the same time, the load disturbance of that same value but of the opposite sign occurs at the bus where the steam turbine 3 in area 2 is connected. It is impossible for MPC to predict this disturbance, however the wind turbine generator could immediately react on this disturbance with its inertial response. A linear model is used for prediction in the MPC algorithm, while the simulations are carried out on a nonlinear model.

Figures $4-6$ present the simulation results on the nonlinear power system model controlled with the linear MPC, and under the above described load disturbance. Only simulation results of area 1 are presented because the simulation results of area 2 are similar. The simulated trajectories represent deviations from the nominal values. The frequency deviation is significantly reduced for both centralized MPC and DMPC, when compared to conventional AGC, which is clearly visible in the upper graph of Figure 4 . Note that in this simulation there hardly exists any difference between centralized MPC and DMPC. Also the tie line power flow is significantly reduced, as can be seen in Figure 6. Centralized MPC gives a slightly better result than DMPC for the tie line power flow. It was supposed that the wind turbine generators are producing $200 \mathrm{~kW}$ under rated power. Figure 5 shows that the constraint value is reached but not exceeded. The response of the wind turbine generator in the case of AGC is absent in Figure 5, because there is no deviation from the nominal operating points in that case. The steam turbine generators are constrained to deviate not more than $500 \mathrm{~kW}$ from their nominal operating point. The bottom graph of Figure 4 shows that the constraint of $500 \mathrm{~kW}$ above nominal power reference input is not exceeded.

Figures $7-9$ present simulation results of a change in PTU setpoints for both areas. The PTU setpoint is increased by $400 \mathrm{~kW}$ for area 1 and decreased by an equal amount for
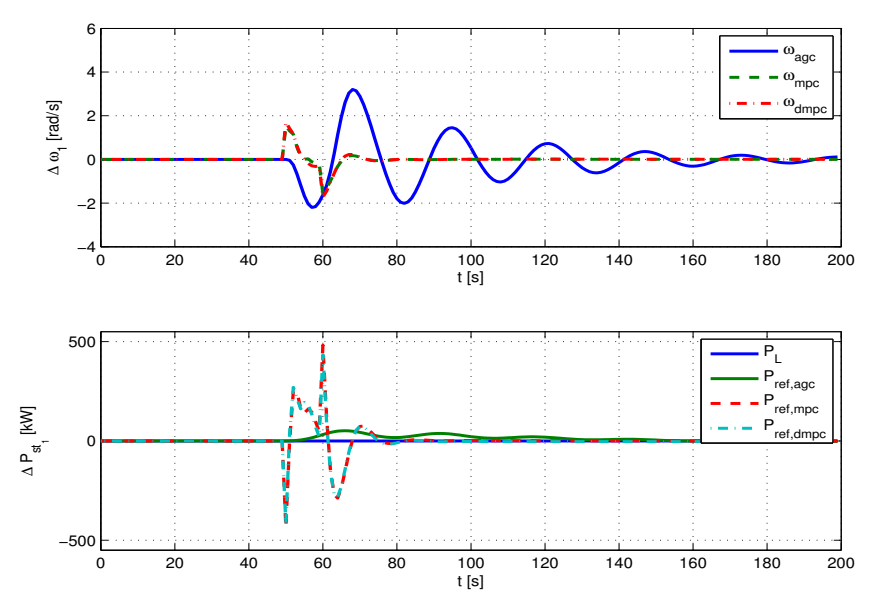

Fig. 4. Response on a load disturbance for steam turbine 1 of area 1.
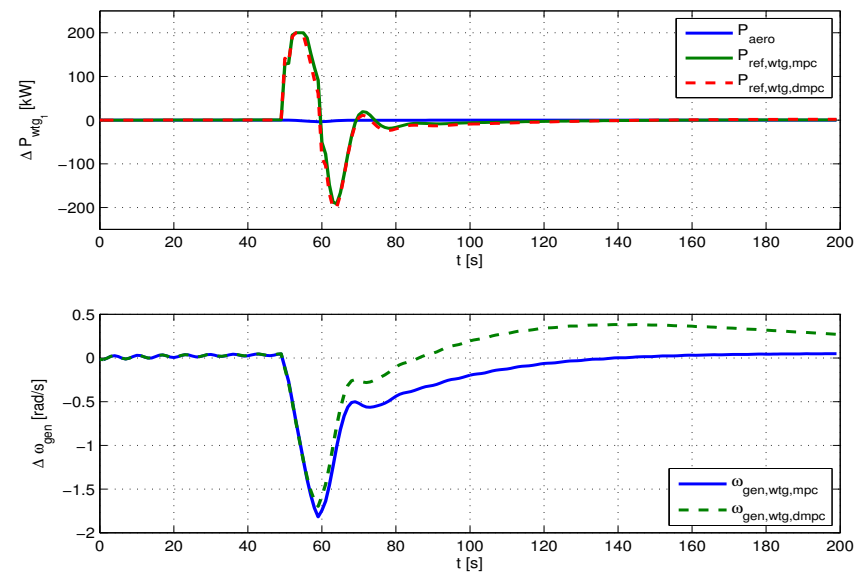

Fig. 5. Response on a load disturbance for wind turbine of area 1.

area 2. For the case of AGC both steam turbine generators in each area are compelled to contribute with an equal amount. When using MPC, the contribution of each generator will be economically dispatched, based on the outcome of an optimization problem. For comparison with AGC both steam turbines of each area contribute with an equal amount. Again a comparison is made between contribution of the wind turbine generator to frequency regulation using a centralized MPC controller, using a DMPC controller and the conventional AGC controller. The weights in the control objective of the MPC controller can be tuned to achieve specific goals. This gives the opportunity to focus more on either frequency deviation reduction or on PTU setpoint tracking. Figure 9 shows a significantly better PTU setpoint tracking using centralized MPC. Although the resulting tie line power flow

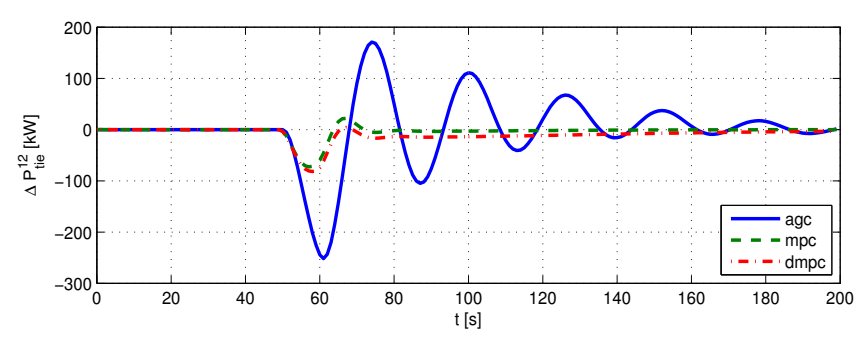

Fig. 6. Tie-line power flow during a load disturbance. 

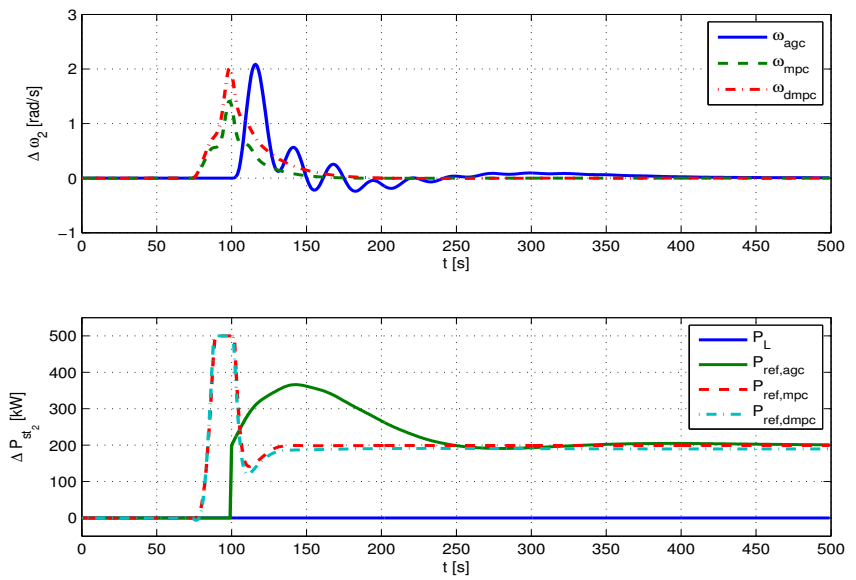

Fig. 7. Response on a PTU boundary for steam turbine 2 of area 1.
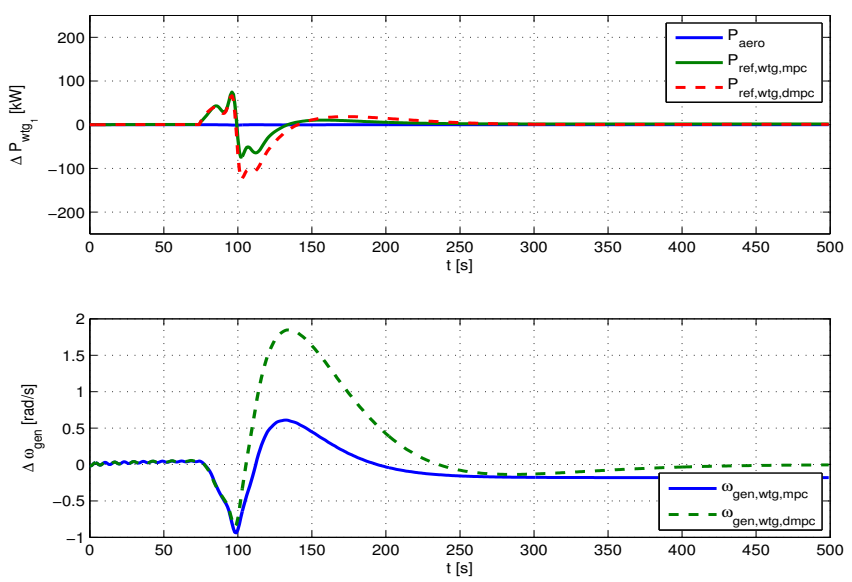

Fig. 8. Response on a PTU boundary for wind turbine of area 1.

of the DMPC controller has no overshoot and is faster than $\mathrm{AGC}$, it has a steady state error. One reason for this is that only the DMPC controller of area 1 is responsible for the tie line power flow between the two areas. The controller of area 1 does not have any influence on the voltage phase angle $\delta_{\mathrm{st}_{3}}$ of the steam turbine generator 3 of area 2, with which the connection with area 1 is established.

Note that in the lower graph of Figure 7 the constraints of the steam turbines are satisfied at all time instances.

The largest benefit derived from using the inertial response of a wind turbine generator is in its effectiveness in compensating for load disturbances. On the PTU boundaries, the benefits can mainly be attributed to the use of MPC. Because PTU setpoints are known beforehand, MPC is able to predict system behavior on the PTU boundaries. The obtained results

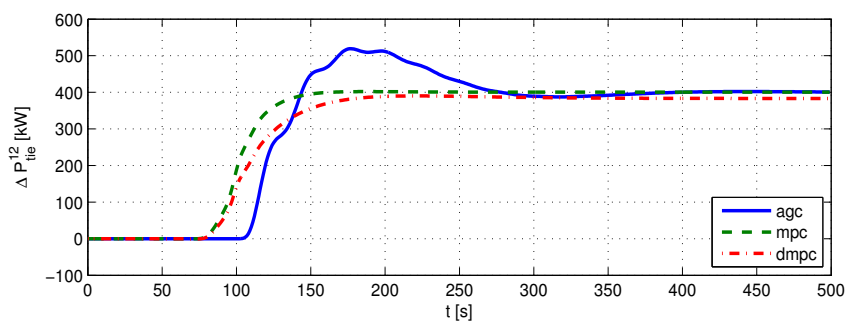

Fig. 9. Tie-line power flow on a PTU boundary. for DMPC are not as efficient when compared to the results for centralized MPC, but present considerable improvement when compared to the classical AGC control.

\section{CONCLUSION}

Wind turbines contain a large amount of kinetic energy in its rotating inertia. This research pointed out that it is technically possible to use this energy to counteract frequency fluctuations in a power grid. Model predictive control approach, presented in this paper, allows for optimal exploitation of this energy in support of real-time system power balancing, while safe operation is guaranteed as safety constraints are explicitly accounted for while computing control actions. To make MPC applicable for large-scale power networks, a decentralized MPC algorithm has been considered in this paper.

\section{REFERENCES}

[1] UCTE Ad-Hoc Group, "Frequency quality investigation report," UCTE, Tech. Rep. Version 10, 2008.

[2] T. Weissbach and E. Welfonder, "High frequency deviations within the european power system: Origins and proposals for improvement," in Proc. Power Systems Conference and Exposition (PSCE '09), Seattle, Washington, 2009.

[3] TenneT, "Operational data," 2009.

[4] European Commission, Directorate-General for Energy, "EU energy trends to 2030," European Commission, Tech. Rep., 2009.

[5] A. Mullane and M. O'Malley, "The inertial response of inductionmachine-based wind turbines," IEEE Transactions on Power Systems, vol. 20, no. 3, pp. 1496 - 1503, aug. 2005.

[6] G. Lalor, A. Mullane, and M. O'Malley, "Frequency control and wind turbine technologies," IEEE Transactions on Power Systems, vol. 20, no. 4, pp. 1905 - 1913, nov. 2005.

[7] J. Morren, S. de Haan, W. Kling, and J. Ferreira, "Wind turbines emulating inertia and supporting primary frequency control," IEEE Transactions on Power Systems, vol. 21, no. 1, pp. 433 - 434, 2006.

[8] A. N. Venkat, "Distributed model predictive control: Theory and applications," Ph.D. dissertation, University of Wisconsin-Madison, 2006.

[9] E. Camponogara, D. Jia, B. Krogh, and S. Talukdar, "Distributed model predictive control," IEEE Control Systems Magazine, vol. 22, no. 1, pp. $44-52$, feb. 2002.

[10] A. C. Damoiseaux, A. Jokić, M. Lazar, P. P. J. van den Bosch, I. A. Hiskens, and A. Bemporad, "Assessment of non-centralized model predictive control techniques for power networks," in Proc. 16th Power Systems Computation Conference (PSCC'08), Glasgow, Scotland, 2008.

[11] B. G. Rawn, P. W. Lehn, and M. Maggiore, "Control methodology to mitigate the grid impact of wind turbines," IEEE Transactions on Energy Conversion, vol. 22, no. 2, pp. 431-438, Jun 2007.

[12] J. G. Slootweg, S. W. H. de Haan, H. Polinder, and W. L. Kling, "General model for representing variable speed wind turbines in power system dynamics simulations," IEEE Transactions on Power Systems, vol. 18 , no. 1 , pp. 144-151, 2003.

[13] B. G. Rawn, "Ensuring safe exploitation of wind turbine kinetic energy: An invariance kernel formulation," Ph.D. dissertation, University of Toronto, 2010.

[14] D. Q. Mayne, J. B. Rawlings, C. V. Rao, and P. O. M. Scokaert, "Constrained model predictive control: Stability and optimality," $\mathrm{Au}$ tomatica, vol. 36, no. 6, pp. 789-814, 2000.

[15] A. Alessio and A. Bemporad, "Decentralized model predictive control of constrained linear systems," in Proceedings European Control Conference, Kos, Greece, 2007, pp. 2813-2818.

[16] P. Kundur, Power System Stability and Control. McGraw-Hill, 1994

[17] G. Michalke, "Variable speed wind turbines - modelling, control, and impact on power systems," Ph.D. dissertation, Technischen Universität Darmstadt, 2008. 